\title{
Akkumulationslogik versus Entwicklungslogik : die Bedeutung der neuen öffentlich-privaten Partnerschaften
}

\section{Frédéric Lapeyre}

\section{OpenEdition}

Electronic version

URL: http://journals.openedition.org/sjep/466

DOI: $10.4000 /$ sjep.466

ISSN: 1663-9677

Publisher

Institut de hautes études internationales et du développement

Printed version

Date of publication: 1 octobre 2005

Number of pages: $25-44$

ISBN: 2-88247-059-2

ISSN: 1660-5926

\section{Electronic reference}

Frédéric Lapeyre, «Akkumulationslogik versus Entwicklungslogik : die Bedeutung der neuen öffentlichprivaten Partnerschaften », Schweizerisches Jahrbuch für Entwicklungspolitik [Online], 24-2 | 2005, Online erschienen am: 08 Juni 2010, abgerufen am 08 September 2020. URL : http://

journals.openedition.org/sjep/466 ; DOI : https://doi.org/10.4000/sjep.466 


\title{
Akkumulationslogik versus Entwicklungslogik: die Bedeutung der neuen öffentlich-privaten Partnerschaften
}

\author{
Frédéric Lapeyre*
}

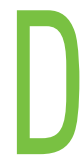

ie ,Truman-Doktrin“, wie sie in Punkt IV der Präsidentenrede über die Lage der Nation von 1949 dargelegt ist, hat die Entwicklungszusammenarbeit tief und dauerhaft geprägt. Truman legte in seiner Rede damals die vorherrschende Sicht der Entwicklung als Modernisierungsprozess aus, der die Umwandlung traditioneller Gesellschaften in moderne Gesellschaften gewährleisten sollte. Diese Dichotomie ,traditionell versus modern“ hat sich in der Folge entscheidend auf den Stellenwert der nichtstaatlichen Akteure und besonders der Akteure des Volkes bei der Festlegung der Entwicklungspolitiken ausgewirkt. Der zentrale Akteur der Entwicklung wird klar definiert: der Staat soll unter der Kontrolle modernisierungsfreundlicher Eliten - die gegen die revolutionären radikalen Eliten unterstützt werden müssen - voluntaristische Wirtschaftsentwicklungspolitiken durchführen. Zu jener Zeit gab es einen weitgehenden Konsens über die Bedeutung der Entwicklungsplanung als wesentliches Instrument sozioökonomischen Wandels.

In diesem Rahmen bestand die Rolle der Entwicklungszusammenarbeit darin, die ,unterentwickelten“ Länder oder Regionen mit finanzieller und technischer Hilfe zu unterstützen, um ihren Wirtschaftsaufschwung zu ermöglichen und den Aufholprozess zu beschleunigen. Die Bevölkerung und besonders die Akteure des Volkes wurden vor diesem Hintergrund als Nichtakteure der Entwicklung gesehen, als Träger traditioneller Werte, die Modernisierungshindernisse waren, als passive und unwissende „Arme“, die auf Hilfe von aussen warteten und denen unbedingt aus der Misere geholfen werden musste. Sie entwickelten sich zu einer Zielbevölkerung der Entwicklungszusammenarbeit und waren in dieser Eigenschaft unaufhörlichen Wellen von Modernisierungs- und Entwicklungspolitiken ausgesetzt, welche sie universalen Modernitätskriterien annähern sollten, die von der historischen Entwicklungserfahrung des Westens ausgehend definiert wurden.

Anfang der 80er Jahre wurde die vorherrschende Sicht der Rolle des Staates in der Entwicklungspolitik durch die neoliberale Gegenrevolution auf den Kopf gestellt. Der öffentliche Sektor verlor seine Position als Entwicklungsmotor zugunsten des - inländischen oder transnationalen - Privatsektors, der die Rückkehr auf den Wachstumspfad gewährleisten sollte. Fortan hatten die Erfordernisse der internationalen Sphäre Vorrang vor jenen der nationalen Sphäre, diejenigen des Privatsektors Priorität vor jenen des öffentlichen Sektors. Die Sensibilisierung für die Exzesse des so genannten „Washington Konsenses“ in

* Professor am Institut für Entwicklungsstudien, Katholische Universität Löwen. 
den 90er Jahren führte zur Rehabilitierung des Staates, der als positive Kraft anerkannt wird, sofern er die Grundsätze guter Regierungsführung verwirklicht. Die vier neuen Hauptprinzipien der Entwicklungszusammenarbeit sind nun Partizipation, öffentlich-private Partnerschaften, Ownership und Empowerment.

Partizipation wird heute als Allheilmittel in der Entwicklungspolitik angepriesen, genau wie die Stukturanpassung und die Liberalisierung in den 80er und 90er Jahren. Die Partizipation bildet ein zentrales Element des ,,integrierten Entwicklungsrahmens" und der Armutsverringerungsstrategien, für die sich die internationale Staatengemeinschaft stark macht (World Bank, 2000). Die Neuausrichtung der Entwicklungszusammenarbeit hin zu nichtstaatlichen Akteuren und die neuen Formen der Konditionalität - Verknüpfung mit der Good Governance förderten in den 90er Jahren den Demokratisierungs- und Dezentralisierungsprozess im Süden. Daraus entstand ein für die Entwicklung neuer Partnerschaften förderlicher Rahmen, der sich in einer Erweiterung des Kompetenzbereichs des Privatsektors, der Organisationen der Zivilgesellschaft und der lokalen Behörden auswirkte.

Zum besseren Verständnis der Tragweite der jüngsten Umwandlungen der Entwicklungszusammenarbeit ist es notwendig, die Meilensteine ihrer Entwicklung Revue passieren zu lassen und die in den letzten Jahren eingeführten neuen Bestimmungen zu analysieren. Diese Bestimmungen - insbesondere das Cotonou-Abkommen und die Strategiedokumente zur Armutsverringerung (Poverty Reduction Strategy Papers, PRSP) - konkretisieren das neue Entwicklungsparadigma, das die Verteilung der Rollen und Verantwortlichkeiten der staatlichen und nichtstaatlichen Akteure grundlegend verändert hat. Abschliessend wird der Akzent auf die Risiken und Chancen des neuen Rahmens der Entwicklungszusammenarbeit für die Volksorganisationen gelegt.

\section{Stellenwert des Staates zu Beginn der Entwicklungszusammenarbeit}

Ein kritischer Blick auf die in den Jahren 1940-1950 entstandene Entwicklungsökonomie zeigt, dass sie von grossem Pessimismus hinsichtlich der Resultate des Laisser-faire der Marktmechanismen, von einer voluntaristischen Vision der Entwicklung und von grossem Optimismus bezüglich der Entwicklungsplanung geprägt war. Das Offenlegen der Unzulänglichkeiten des Marktes rechtfertigte die direkte staatliche Intervention im Produktionsbereich, nachdem die Koordinierung und die Verwaltung des öffentlichen Sektors nicht als problematisch beurteilt wurden (Tinbergen, 1984 : 326). Die bis in die frühen 70er Jahre massgebliche normative Theorie des Staates sah somit vor, dass der Staat Mängel des Marktes mit angemessenen Interventionen beheben und dadurch das Wohlergehen der Gemeinschaft insgesamt verbessern sollte.

Die Entwicklungszusammenarbeit wurde von dieser durchweg optimistischen Sicht der staatlichen Intervention stark geprägt, die jedoch drei bedeutende Lücken aufwies: erstens unzureichende Überlegungen zum Problem der Interaktion Staat-Markt im Rahmen einer gemischten Wirtschaftsordnung (es wird verkannt, dass die jeweiligen Stärken und Schwächen effizient kombiniert werden müssen); zweitens ein vereinfachendes Konzept der Natur des Staates und der Auswirkungen staatlicher Politik; drittens die Nichteinbeziehung der Zivilgesellschaft in den Entscheidungsprozess. Genau in diese Breschen springen 
später die neoliberalen Ökonomen und andere, um die Rolle des Staates in der Entwicklung zu unterlaufen.

\section{Die neoliberale Gegenrevolution und ihre Folgen}

Der politische und akademische Sieg der neoliberalen Denkrichtung in den 80er Jahren äusserte sich in vielen Kreisen, die sich mit Entwicklungspolitik beschäftigen, in einer grundlegend veränderten Wahrnehmung des Staates. Die neue Wirtschaftspolitik (NEP) ersetzte die von den Pionieren der Entwicklungszusammenarbeit aufrechterhaltene, etwas naive Vision eines freiwillig engagierten Staates durch die äusserst zynische Vision eines raubgierigen und korrupten Staates. In diesem Rahmen versucht jede spezifische Interessengruppe - Politiker, Wähler, Beamte, Gewerkschaften, inländische Produzenten, usw. - den Staat für ihre Zwecke einzuspannen und auf Kosten der gesamten Gesellschaft maximale eigene Vorteile herauszuschlagen. Die ganze Aufmerksamkeit konzentrierte sich auf das Problem des zunehmenden Gewichts des Staates auf die Wirtschaft, der durch die Stimulierung des Konkurrenzkampfes um die Einkommen der privaten wie auch der öffentlichen Wirtschaftssubjekte als der Ursprung aller Verzerrungen gesehen wurde, unter denen sowohl die keynesianischen Staaten des Nordens als auch die voluntaristischen Staaten des Südens und die sozialistischen Systeme im Osten litten (Krueger, 1990 : 426).

Die kontraproduktiven Auswirkungen der staatlichen Intervention wurden angeprangert: irrationaler und kostspieliger öffentlicher Sektor, Verzerrungen in den Produktions- und Verteilungsstrukturen, ineffiziente dirigistische Preispolitik, usw. Die Vertreter des Neoliberalismus argumentierten, dass die Kosten des Interventionismus gegenüber den Vorteilen überwiegen würden, und dass im Zweifelsfall die Mängel des Marktes geringeren Schaden anrichten würden als die Mängel des Staates. So wurde jeder Versuch des Staates, das Armutsproblem zu beseitigen, von der NEP wegen der kontraproduktiven Auswirkungen seiner Intervention unweigerlich als eine Verschlimmerung des Armutsproblems angesehen. Der Voluntarismus des Staates in der Wirtschaft führte gemäss der NEP zu einer Entwicklung der legalen und illegalen Wirtschaftstätigkeiten, die dazu bestimmt waren, der durch die öffentliche Verwaltungstätigkeit erzeugten Einkommen habhaft zu werden. Der Staat sollte demnach nicht mehr als für alles zuständig und um die Maximierung des Gemeinwohls bemüht, sondern als Resultat einer komplexen Wechselwirkung zwischen Interessengruppen angesehen werden. So wurde eine vereinheitlichte Theorie der menschlichen Motivationen zur Diskussion gestellt, wonach die rationellen Entscheidungen der individuellen Wirtschaftssubjekte - des öffentlichen wie auch des privaten Sektors - der Hypothese der Maximierung ihres persönlichen Interesses folgen (Tollison, 1982).

Die neue dominierende Denkströmung schlug die Lösung vor, den Staat auf seine Mindestgrösse zu reduzieren und die Ressourcenallokation wieder dem freien Spiel der Marktmechanismen zu überlassen. Es ist offensichtlich, dass die NEP die Strukturanpassungs- und Reformprogramme, für welche die internationalen Institutionen in den 80er und 90er Jahren plädierten, weitgehend geprägt hat. Der 1981 veröffentlichte Berg-Bericht der Weltbank ist ein Lehrbuchbeispiel dafür: Die Entwicklungskrise in Afrika wird auf die übermässige staatliche Intervention zurückgeführt, die zu einer Unterentwicklung der Landwirtschaft 
und des Exportsektors geführt habe (Weltbank, 1981). Das Leitmotiv des Berichts - dessen Grundphilosophie jener der NEP ähnlich ist - betrifft die Ineffizienz und die Kosten des Staates in Afrika. Das setzt auf der Ebene der volkswirtschaftlichen Reform voraus, dass der Staat reduziert wird und die Managerkapazitäten des Privatsektors entwickelt und unterstützt werden.

\section{Strukturanpassungsprogramme und Neudefinition der Rolle des Staates}

Eines der Hauptziele, das die internationalen Organisationen mit den Strukturanpassungsprogrammen verfolgten, war, eine Neuverteilung der Rollen zwischen dem privaten und dem öffentlichen Sektor durchzusetzen (Jolly, Emmerij, Ghai und Lapeyre, 2004). Der Staat sollte den öffentlichen Sektor durch die Neubewertung seiner Tätigkeiten nach Kriterien ordentlicher Verwaltungsführung und effizienter Allokation knapper Ressourcen rationalisieren. Die Doktrin der Strukturanpassung propagiert insbesondere eine wichtigere Rolle des Preissystems bei der Allokation der wesentlichen Dienstleistungen (Ausbildung, Gesundheit, Verkehr, Wasser, Energie, usw.), da diese sich in der Hand des Staates als eine Quelle von Kosten und Verschwendung herausgestellt haben; aus diesem Grund wird ihre Privatisierung befürwortet (Colclough, 1992 : 199). Zum anderen sollte die Verringerung der halböffentlichen Tätigkeiten besonders in den direkten Produktionstätigkeiten und den potenziell profitablen Subsektoren wie Handel und Verkehr - Möglichkeiten für die dynamischen und flexiblen Unternehmer des Privatsektors erschliessen.

Bei dem neuen Wachstumsmodell sollte der Staat keine auf einer nationalen Entwicklungsstrategie beruhenden voluntaristischen Politiken mehr durchführen, weil diese zum Misserfolg geführt hatten. Der Schwerpunkt lag auf der Reduzierung des öffentlichen Sektors und der öffentlichen Investitionen zugunsten der Stimulierung der Marktkräfte durch eine Reihe umfassender Massnahmen, die den privaten Akteuren das für ihre Tätigkeiten günstigste sozioökonomische Umfeld bieten sollten. Der Staat sollte die Wirtschaft liberalisieren und sich aus seinen traditionellen Tätigkeiten zurückziehen, ausgenommen im Fall der für den Privatsektor bestimmten Wirtschaftsinfrastrukturen. Die Verantwortung des Staates für den maximalen Zugang zu den wesentlichen Grunddiensten wurde im Namen der wirtschaftlichen Rationalität in Frage gestellt, welche die Privatisierung eines Grossteils dieser Dienstleistungen und die Anhebung der Tarife erforderte, unabhängig davon, ob sie privatisiert worden waren oder nicht.

Erwähnenswert in dieser Hinsicht ist der ausgeprägt normative Tenor des Diskurses über die Dezentralisierung und die Förderung der dezentralisierten Akteure (dazu gehört auch der herrschende Diskurs über die Förderung des informellen Sektors). Diese Ziele stimmen mit der Ende der 70er Jahre lancierten neoliberalen Offensive der Delegitimation des Staates und der Auflösung der nationalen Solidarität überein. Der Entflechtungsprozess und die Dezentralisierung haben die Fähigkeit der Staaten geschwächt, nationale Entwicklungspolitiken durchzuführen. Der Staat als Garant des sozialen Zusammenhalts und der Raumplanung im weiteren Sinne wird in Frage gestellt und seine Rolle ausgehend von den Sachzwängen der Logik der leistungsstarken Pole und der Bewältigung der damit verbundenen sozialen und räumlichen Fragmentierungsprozesse wird neu definiert (Peemans, 1997). 
Der Abbau des öffentlichen Sektors, der Rückgang der formellen Beschäftigung (als Ergebnis der Privatisierungen und der Öffnung der Wirtschaft für den internationalen Wettbewerb) sowie die restriktive Haushaltspolitik haben die Befürworter des nationalen Modernisierungsprojektes zugunsten der sozialen Schichten geschwächt, die in der Umsetzung der Strukturanpassungsprogramme das Mittel zur Steigerung ihrer Macht sahen. Die Strukturanpassungsprogramme betrachteten diese Schichten als positive Wachstumskräfte und trugen somit dazu bei, die Eliten des Südens, die dem Privatsektor nahestehen und die Integration in die Weltwirtschaft befürworten, zu stärken. Auch der Präsident der Weltbank, James Wolfensohn, anerkannte den neuen Kontext, indem er erklärte, ein weiterer grosser Wandel sei erfolgt. Die Arbeitgeber, die Investoren oder der Privatsektor belegten heute einen ganz anderen Platz als vor sieben Jahren. Der Privatsektor sei damals nur halb so gross gewesen wie der öffentliche Sektor, während er heute 240 Milliarden Dollar, also das Fünffache des öffentlichen Sektors ausmache. Dabei handle es sich nicht nur um eine quantitative Veränderung, sondern auch um einen qualitativen Sprung. Im Entwicklungsbereich gebe es einen neuen, gewichtigen Akteur (Wolfensohn, 1997: 2).

\section{Das neue Entwicklungsparadigma in den 90er Jahren}

Ende der 80er Jahre zeichnete sich ein breiter Konsens über die dramatischen sozialen Kosten der Strukturanpassungsprogramme und ihre bezüglich der Rückkehr zum Wachstum enttäuschenden Resultate ab. Die Publikation des Buches Adjustment with a Human Face durch die UNICEF im Jahr 1980 und dann die Verbreitung von Fragen menschlicher Entwicklung in den anderen internationalen Entwicklungsorganisationen markierten das Aufkommen des neuen Konsenses zur Armutsbekämpfung, der sich zum Kernpunkt des dominanten Diskurses in den 90er Jahren entwickeln sollte (Cornia, Jolly und Stewart, 1987; UNDP, 1990; World Bank, 1990).

Im Rahmen dieser Entwicklung ist besonders die Präsentation von Joseph Stiglitz im Jahr 1998 anlässlich des von der UNCTAD organisierten Konferenzzyklus Raúl Prebisch zu erwähnen. Darin kommt der „Post-Washington-Konsens" zum bevorstehenden sozioökonomischen Umwandlungsprozess sehr klar zum Ausdruck. Der Konsens beruhte auf vier Hauptelementen: Verbindung zwischen Markt und Staat mit dem Privatsektor als Wachstumsmotor, Integration in die globalisierte Wirtschaft, Demokratisierung und gute Regierungsführung.

Stiglitz hat 1999 im Namen der Weltbank ein wahrhaftes Schuldbekenntnis für die in den 80er Jahren auferlegte Form der Konditionalität abgelegt. Die internationalen Organisationen gestanden aufgrund interner Evaluationsberichte zu den von ihnen finanzierten Entwicklungsprojekten und -programmen ein, dass jeder Versuch, Veränderungen von aussen aufzuzwingen, grosse Gefahr laufe, auf inneren Widerstand zu stossen und anstatt den Wandel zu fördern, eine Abwehrhaltung hervorzurufen (World Bank, 1998; Isham, Narayan und Pritchett, 1995). Infolgedessen soll die Einführung von Partizipationsmechanismen bei der Gestaltung der Entwicklungsstrategie erlauben, einen Konsens hinsichtlich der ausgearbeiteten Strategie zu entwickeln und dadurch einen Prozess der Aneignung der Reformen durch die Bevölkerung zu fördern, um ihre Erfolgschancen zu steigern und die Risiken sozialer und politischer Instabilität zu verringern (Stiglitz, 1998: 14). 
Die jüngsten Erklärungen Wolfensohns sind in dieser Hinsicht repräsentativ: Laut Wolfensohn ist die Entwicklung keine Frage der Nächstenliebe, sondern eine Frage der Einbeziehung und des Kapazitätsaufbaus. Die unter der Armut leidenden Bevölkerungsgruppen wollen mitreden. Zudem sei die Partizipation eine Gewährleistung für greifbare Ergebnisse in Bezug auf die Projekte und Programme. Sie könne den Konsens herbeiführen, der die Grundlage der sozialen Veränderungen und Reformen bildet (Wolfensohn, 2000). Der Weltentwicklungsbericht 2000/2001 der Weltbank mit dem Titel Combating Poverty übernimmt explizit die Idee des Empowerment als entwicklungsinhärenter Dimension und als Mittel zur Armutsbekämpfung.

Das wachsende Interesse für das Konzept des sozialen Kapitals in den 90er Jahren hängt auch mit der Anerkennung der grundlegenden Rolle der Zivilgesellschaft und den mehr oder weniger institutionalisierten sozialen Mediationen zwischen Staat, Markt und Zivilgesellschaft im Entwicklungsprozess zusammen (Woolcock, 1998: 155). Das Konzept des sozialen Kapitals verweist auf den Bestand von Normen der Gegenseitigkeit und des Vertrauens sowie auf Netzwerke bürgerlichen Engagements als Merkmale der sozialen Organisation (Putnam, 1993a: 167). Diese können durch die Förderung abgestimmter Aktionen zu einer effizienteren Gesellschaft beitragen, weshalb der Bestand an sozialem Kapital, von dem das Ausmass der Zusammenarbeit in der Gesellschaft abhängt, sehr wichtig ist. Die Berücksichtigung des sozialen Kapitals neben dem Sachund Humankapital geht auf die Erkenntnis zurück, dass der Bestand an sozialem Kapital einer Gemeinschaft für das Lancieren kohärenter, effizienter und tragfähiger Entwicklungsinitiativen bedeutsam ist. Die Schaffung eines entwicklungsförderlichen Umfelds ist das Ergebnis all dieser sozialen Bande, die den Staat an die Gesellschaft binden, und bietet institutionalisierte Kanäle an, die kontinuierliche Verhandlungen über Ziele und Politiken ermöglichen (Evans, 1995: 164).

Gemäss der liberalen Denkweise eignet sich die staatliche Intervention nicht dazu, das soziale Kapital zu steigern, sondern führt im Gegenteil zur Vernichtung dieses Kapitals (Coleman, 1990; Fukuyama, 1995). Ein Grossteil der Literatur über das soziale Kapital legt indessen den Nachdruck auf die Idee von Synergien. Die Annahme von Synergien setzt voraus, dass der Staat zu einem dem Engagement der Zivilgesellschaft im Entwicklungsprozess förderlichen Umfeld beiträgt, und zwar durch die Schaffung eines Vertrauensklimas und die Stimulierung von Netzwerken der betroffenen Akteure, deren Mobilisierung im Gegenzug die Effizienz und Tragfähigkeit der Entwicklungspolitiken gewährleistet (Putnam, 1993b: 42). Evans hebt das Entwicklungspotenzial einer Kombination aus starken öffentlichen Institutionen und organisierten Gemeinschaften hervor, bei der die Komplementaritäten aller optimal genutzt werden können (Evans, 1996 : 130). Der Staat spielt bei der Reproduktion des sozialen Kapitals eine Schlüsselrolle, weil diese von allen Institutionen abhängt, die den Austausch fördern und das Kooperationsniveau steigern sollen. Somit handelt es sich hier eindeutig um ein „Positivsummenspiel“. 
Die traditionelle Verantwortung des Staates bestand darin, den Zugang der Bevölkerung zu den wesentlichen Dienstleistungen zu gewährleisten. Jedoch ist im Süden seit über zwei Jahrzehnten eine wachsende Rolle des Privatsektors im Gesundheits- und Erziehungswesen sowie eine Tendenz zur Privatisierung der Wasser- und Stromversorgung und der Abwasserentsorgungsdienste zu beobachten. Für diese Entwicklung werden drei Gründe angeführt: geringe öffentliche Ressourcen, mittelmässige Qualität des öffentlichen Dienstes, Druck zugunsten der Wirtschaftsliberalisierung. Dabei steht der Gedanke im Vordergrund, dass eine öffentliche Dienstleistung nicht immer die optimale Lösung darstellt, zumal wenn die Institutionen schwach sind und die öffentlichen Mittel in untransparenter Weise eingesetzt werden (UNDP, 2003). Die Bretton-Woods-Organisationen weisen deshalb unter anderem auf das Problem hin, dass allzu viele öffentliche Dienste von den verantwortlichen staatlichen Behörden schlecht verwaltet werden. Ausserdem kann der Staat in zahlreichen armen Ländern wegen akuten Ressourcenmangels weder effiziente und universale Gemeinschaftsdienste gewährleisten, noch bedeutende Infrastrukturinvestitionen für die Wartung und den Ausbau dieser Dienste finanzieren. Diese Verhältnisse behindern die Verwirklichung der Millenniums-Entwicklungsziele der Vereinten Nationen wesentlich, darunter insbesondere das Ziel, den Anteil der Menschen ohne Zugang zu sauberem Trinkwasser bis zum Jahr 2015 zu halbieren.

Aus diesem Grund appelliert die Weltbank systematisch an die Staaten, sich um einen effizienteren Einsatz der öffentlichen Mittel und ein besseres Preis-Leistungs-Verhältnis der unentbehrlichen Dienstleistungen zu bemühen. Die Verfolgung dieser Ziele hat entweder zur Privatisierung oder zu einer radikalen Änderung des öffentlichen Verwaltungsmodus dieser Dienstleistungen geführt. Zum einen ermöglichte die Privatisierung die Abschaffung der Subventionen an die defizitären staatlichen Betriebe, welche die öffentlichen Ausgaben belasten (Priorität der Bretton-Woods-Institutionen), da bei den öffentlichen Infrastrukturen die Tarife oft zu niedrig sind, um die Kostendeckung zu gestatten, und Probleme mit Zahlungsausfällen gang und gäbe sind. Die Privatisierung sollte ausserdem die Effizienz, die Produktivität und die Rentabilität im Sektor der wesentlichen Dienstleistungen verbessern, indem die Rationalität und die Dynamik der Marktkräfte in die Beschlussfassung und die Geschäftsführung eingeführt werden. Zum anderen beruhen die neuen Vorschriften öffentlicher Verwaltung auf dem Grundsatz der totalen Kostendeckung, selbst wenn diese Dienstleistungen nicht privatisiert wurden. Dieser Grundsatz schreibt es den Dienstleistungserbringern vor, ein Einkommensniveau anzustreben, das die Deckung aller ihrer regelmässig wiederkehrenden Ausgaben ermöglicht, bei gleichzeitiger Ausarbeitung einer nachhaltigen Kostendeckungspolitik, die den künftigen Finanzbedarf berücksichtigt (Camdessus, 2003 : 24). In beiden Fällen - Privatisierung und neue öffentliche Verwaltung - wurden die ärmsten Bevölkerungsgruppen mit einem starken Kostenanstieg und sich verschärfenden Zugangsproblemen konfrontiert.

In den 80er und 90er Jahren griffen die neuen herrschenden Eliten die Forderungen der Marktkräfte betreffend die Wirtschaftsliberalisierung und die Öffnung der Märkte auf, welche zum Aufschwung privater Erbringer für die wesent- 
lichen kollektiven Dienste führten. Die Geldgeber spielten ebenfalls eine wichtige Rolle in diesem Sinne, indem sie verlangten, dass auch diese Dienste besonders die Wasserversorgung in den Städten - privat erbracht und finanziert werden sollten. Auch das Allgemeine Abkommen über den Dienstleistungshandel (GATS) der WTO fördert eine teilweise Privatisierung. Die Länder müssen sich im Rahmen dieser Abkommen zu einer „fortschreitenden Liberalisierung“ verpflichten und werden immer eindringlicher aufgefordert, neue Aspekte der Dienstleistungserbringung zu liberalisieren.

Es ist zu betonen, dass die Häufung der öffentlich-privaten Partnerschaften keineswegs das logische und unvermeidbare Resultat der Rückkehr zur ökonomischen Rationalität der Marktkräfte darstellt. Diese Partnerschaften wurden in den letzten Jahren von den dominierenden Akteuren im Rahmen der mit dem ordnungsgemässen Ablauf der globalisierten Wirtschaft beauftragten neuen globalen Architektur institutionalisiert, geschützt und gelenkt. Im Jahr 2002 stellten die Konferenz der Vereinten Nationen über die Entwicklungsfinanzierung von Monterrey und der Weltgipfel der Vereinten Nationen über die nachhaltige Entwicklung von Johannesburg diese neuen Partnerschaften in den Mittelpunkt der Umwandlungen der Entwicklungszusammenarbeit, indem sie sie zu einer der verheissungsvollsten neuen Perspektiven hinsichtlich der Auswirkungen auf die Lebensbedingungen der Bevölkerung machten.

Die Weltbank hat zusammen mit anderen internationalen Institutionen innovative Vorkehrungen getroffen, um dem Privatsektor bei der Verwaltung der wesentlichen Dienstleistungen eine wachsende Rolle einzuräumen. Die Garantien der Weltbank, die Internationale Finanzgesellschaft und die Multilaterale Investitionsgarantie-Agentur helfen so den Regierungen, Privatinvestoren durch die Verringerung der mit der Leistung der Regierungen verbundenen Risiken in den Wasserversorgungs- und Abwasserentsorgungssektor anzuziehen. Die Weltbank gewährt ausserdem Darlehen für die Finanzierung von Projekten, welche die Auflagen der Privatisierung und der Kostendeckung erfüllen. Das trifft auf das Beispiel des Wasserversorgungsprojektes in Luanda, Angola, zu, das namentlich Privatunternehmen für die Verwaltung des Wasserversorgungssystems in den wichtigsten urbanen Zentren anwerben und ein Tarifsystem einführen soll, das die Kostendeckung durch die Wasserversorgungsgesellschaft erlaubt.

Die neue vorherrschende Sicht der Verwaltung wesentlicher Dienste prägt auch die im Jahr 2001 gegründete Neue Partnerschaft für die Entwicklung Afrikas (NEPAD), welche Partnerschaften zwischen dem öffentlichen und dem privaten Sektor fördert, um in verschiedenen lebenswichtigen Sektoren, darunter die Wasserversorgung, neue Investitionen anzuziehen. Diese Position markiert bei den herrschenden afrikanischen Eliten einen tief greifenden Wandel. Sie setzen auf die Transnationalisierung, indem sie den ausländischen Direktinvestitionen der multinationalen Konzerne Tür und Tor öffnen und ihnen Möglichkeiten zur Kapitalwertsteigerung in neuen Tätigkeitsbereichen (Transport, Energie, Wasser und Abwasserentsorgung) bieten.

Heute werden in den Sektoren der Wasserversorgung und der Abwasserentsorgung über 2350 öffentlich-private Partnerschaften gezählt, während sie Anfang der 90er Jahre praktisch inexistent waren (UNDP, 2003 : 116). Die Förderung der Partnerschaften und die Neufestlegung der Zuständigkeitsbereiche des öffentlichen und des privaten Sektors werfen jedoch Probleme auf, da diese 
Politiken - unter dem Deckmantel der Verbesserung der Qualität und des Zugangs zu den wesentlichen Dienstleistungen - zu manchmal umstrittenen Ergebnissen führten, wenn sie mit der Privatisierung der Dienstleistungen verbunden waren. Das Problem stellt sich immer dann, wenn die privaten Gesellschaften die Wasserversorgung ländlicher Gebiete oder einfacher Quartiere in einkommensschwachen Ländern verweigern, da sie diese Aktivität aufgrund des Fehlens solventer Märkte in diesen Gebieten für unrentabel halten (Saprin, 2004). In Cartagena, Kolumbien, wurde zum Beispiel ein grosses Slumgebiet nicht ans Netz angeschlossen, weil der Dienstleistungserbringer der Ansicht war, dass es nicht zur Agglomeration gehöre.

Somit weisen diese Partnerschaften gemischte Ergebnisse auf. Entgegen den Erwartungen konnte das erhoffte frische Kapital nicht mit der Privatisierung beschafft werden, und die neuen öffentlich-privaten Partnerschaften konnten diese zusätzlichen Ressourcen zur Verbesserung der Qualität und des Zugangs zu den wesentlichen Diensten nicht mobilisieren. Ausserdem leiden die Ärmsten unter der Weigerung der Privatbetreiber, wenig rentable Investitionen in ärmeren Gebieten zu finanzieren, und sind mit dem Prinzip, dass der Kunde für die Dienstleistung zahlt, konfrontiert, auf dem die öffentlich-privaten Partnerschaften beruhen. In der bolivianischen Stadt Cochabamba beispielsweise ist im Jahr 2000 der Wasserpreis nur einige Wochen, nachdem ein Londoner Privatunternehmen den Zuschlag für die Wasserversorgung der Stadt erhalten hatte, um das Drei- bis Vierfache angestiegen (UNDP, 2003 : 117).

Diese Probleme zeigen die Grenzen der öffentlich-privaten Partnerschaften auf, wenn das Konzept des universalen Zugangs zu den wesentlichen Diensten Geltung behalten soll. Die Sensibilisierung für das Risiko, dass die ärmeren Bevölkerungsschichten vom Zugang zu den wesentlichen Diensten ausgeschlossen werden könnten, liegt den Überlegungen über die globalen öffentlichen Güter zugrunde. Diese sind keine beliebigen Güter und müssen deshalb entweder über Mechanismen, die nicht der Marktlogik gehorchen, oder aber über veränderte Marktmechanismen geliefert werden, die gewährleisten, dass niemand ausgeschlossen wird (Kaul, Grunberg und Stern, 1999). Das Aufkommen des Konzepts sozialer Unternehmensverantwortung war eine Reaktion auf die wachsende Besorgnis über diesen umfassenden Prozess der Privatisierung wesentlicher Dienstleistungen. Es bedeutet, dass eine neue Art von Verantwortung, eine soziale Verantwortung, einbezogen wird, und dass das Unternehmen nicht länger nur als Wirtschaftseinheit betrachtet wird, die sich allein der Gewinnmaximierung verschreibt, sondern auch als ein Stakeholder unter anderen in seinem Umfeld, der es sich schuldig ist, zur Verbesserung der Lebensund Arbeitsbedingungen der Bevölkerung beizutragen, indem er dabei gleichzeitig seine Rentabilität aufrechterhält (Hopkins, 2004).

Allerdings ist bezüglich dieser konflikt- und geschichtslosen Vision der sozialen Errungenschaften Vorsicht geboten. Ihr Schwerpunkt liegt auf dem rein freiwilligen und unverbindlichen Charakter der Initiativen sozialer Unternehmensverantwortung, wohingegen die Geschichte lehrt, dass die sozialen Errungenschaften stets das Ergebnis eines zähen Ringens waren. Die soziale Verantwortung der Unternehmen darf nicht an die Stelle der nationalen und internationalen Regelungen und Gesetze über soziale und ökologische Rechte treten, da sonst die Gefahr der Verunsicherung der Lebens- und Arbeitsbedingungen der Bevölkerung droht. 
Ein starker öffentlicher Wille ist daher erforderlich, um zu verhindern, dass die Privatisierung auf Kosten der Ärmsten erfolgt. In Bolivien wurden die Wasserversorgungs- und Abwasserentsorgungsdienste in La Paz und in El Alto beispielsweise demjenigen Anbieter zugeschlagen, der die grösste Anzahl Anschlüsse in den Armenvierteln in Aussicht stellte.

\section{Neudefinition der Rolle der Bevölkerung und ihrer repräsentativen Organisationen bei der Erbringung wesentlicher Dienstleistungen}

Die Erbringung kollektiver Dienstleistungen durch NGO wird auch als einen bewährten Mittelweg zwischen Markt und Staat betrachtet, um die Lücken des öffentlichen Systems zu schliessen und den Armen den Zugang zu den wesentlichen Diensten zu ermöglichen. Deshalb verzeichnen die Partnerschaften zwischen öffentlicher Hand, Unternehmen und Organisationen der Zivilgesellschaft eine starke Zunahme. Die Beteiligung der NGO verbessert das Verständnis für die Bedürfnisse der armen Gemeinschaften. Ausserdem sind die NGO in der Lage, über Sensibilisierungs- und Mobilisierungskampagnen Druck auszuüben, damit die Privatunternehmen diese Bedürfnisse berücksichtigen.

Im jüngsten Bericht des Generalsekretärs der Vereinten Nationen über die Zivilgesellschaft kommt diese neue, auf der Förderung der öffentlich-privaten Partnerschaften beruhende Vision der Entwicklung anschaulich zum Ausdruck. Der Leitgedanke besteht darin, dass die potenziellen oder existierenden Synergien zwischen den verschiedenen Akteuren der Entwicklung genutzt werden sollen und Kontakte zwischen den verschiedenen von einem Problem betroffenen beteiligten Parteien geknüpft werden müssen (United Nations, 2004). Dank der Abstimmung bei der Zielermittlung und der Projektdefinition wird das Projekt verbessert, bereichert, fester in der Realität verankert und mit den Entwicklungswünschen der Bevölkerung in Einklang gebracht. Die Neudefinition der Rollen und Verantwortungen der (nationalen oder lokalen) öffentlichen Akteure und der privaten Akteure (Privatsektor oder Zivilgesellschaft) soll ferner dazu beitragen, die Verwaltung einer ganzen Reihe wesentlicher Dienstleistungen zu verbessern, da sich, wo Kapazitäten in den Dienst von Multi-Stakeholder-Prozessen gestellt werden, beträchtliche positive Folgen ergeben (United Nations, 2004 : 11). Diese Partnerschaften bilden fortan ein sehr wichtiges Instrument für die Entwicklungszusammenarbeit, um innovierende Antworten auf kritische Fragen zu finden. Die Millenniums-Entwicklungsziele haben auch den Partnerschaften vor Ort zwischen den staatlichen Behörden, dem Privatsektor und den NGO neuen Elan verliehen, da die Verwirklichung dieser Ziele zwingend eine abgestimmte und vielseitige Reaktion erfordert.

Vor diesem Hintergrund ist eine wachsende Beteiligung der NGO in den zwischenstaatlichen Organen und den verschiedenen Entwicklungsprogrammen und -projekten $\mathrm{zu}$ beobachten. Die Tragfähigkeit der Multi-Partnerschaften zeigte sich in der Vielzahl der lokalen Initiativen und im Erfolg zahlreicher Initiativen, denen es gelang, komplexe Probleme zu lösen und schwierige Ziele zu erreichen, indem sie in jeder Phase der Initiative eine Vielzahl von Akteuren mobilisierten. Nachdem der Erfolg eines Grossteils dieser neuer Partnerschaften anerkannt wurde, wurden die nichtstaatlichen Akteure nicht länger als von einer zwischenstaatlichen oder staatlichen Institution beauftragte einfache Ausfüh- 
rungsträger, sondern als vollwertige Partner bei der Ausarbeitung der Politiken und der Beschlussfassung angesehen.

Die Ausdehnung der Entwicklungszusammenarbeit auf eine breite Palette von Akteuren - inbesondere Akteure der Zivilgesellschaft sowie Lokalbehörden und Parlamentarier - wird als Gelegenheit gesehen, neue Kapazitäten und unterschiedliche Erfahrungen zu mobilisieren, um einige der schwierigsten Probleme unserer Zeit in Angriff zu nehmen (Cardoso, 2004 : 8). Die Kommission Cardoso hat dazu eindeutige Empfehlungen formuliert. Die UNO sollte den Schwerpunkt auf die Einbeziehung aller betroffenen Parteien legen und gleichzeitig erkennen, dass die Schlüsselakteure nicht in allen Fällen die gleichen sind. Deshalb ist es wesentlich, Partnerschaften mit verschiedenen Parteien zu fördern, um effiziente Lösungen zu finden und es einer Reihe globaler Netzwerke zu erlauben, zu innovieren und Dinge in Bewegung zu setzen (Cardoso, 2004 : 19).

Der Prozess der Demokratisierung und der Öffnung der Entwicklungszusammenarbeit hin zur Zivilgesellschaft ging mit einem breiten Dezentralisierungsprozess im Süden einher, der darauf abzielte, die lokalen öffentlichen Behörden als Entwicklungsakteure zu stärken. Sie gelten heute als Schlüsselpartner, weil sie in der Regel auf lokaler Ebene eine grundlegende Rolle in für die Lebensbedingungen der Bevölkerung wesentlichen Bereichen wie Wasserversorgung, Abwasserentsorgung, Gesundheits- und Erziehungswesen spielen. Es wird empfohlen, neue lokale staatliche Organe zu fördern, da diese die notwendigen Investitionen häufig viel billiger als der Privatsektor tätigen können, insbesondere indem sie nicht importieren, sondern vor Ort einkaufen.

Die neue Weltordnungspolitik muss deshalb von der Förderung der Partizipation und der Verantwortung auf allen Ebenen ausgehend aufgebaut werden. Das Engagement der Beteiligten und die Entwicklung der Partnerschaften erfordern jedoch einen Kapazitätsausbau der Organisationen der Zivilgesellschaft und des Privatsektors, Massnahmen zum Erreichen von Kompromissen, zu denen auch Mechanismen zur Konfliktprävention und -beilegung zählen, sowie die Ausbildung von Beamten auf nationaler und multinationaler Ebene für den Umgang mit einer breiteren Palette von Beteiligten. Hierfür muss ein institutionelles Umfeld geschaffen werden, das diese neuen Partnerschaften hervorbringen kann und den verschiedenen Beteiligten die Mittel gibt, zu handeln und ihr Potenzial besser auszuschöpfen.

Die Vielzahl der Akteure erscheint fortan als notwendige Voraussetzung für die Entwicklungspolitik, jedoch warnt die Kommission Cardoso davor, in den Partnerschaften ein Allheilmittel für alle Probleme zu sehen. Der Begriff „Partnerschaft" ist unscharf und wird zwangsläufig viele Probleme hervorrufen, da er ein Ausmass von Gleichheit unter den Beteiligten voraussetzt, das in der Praxis selten existiert. Zum anderen stellt sich die Frage der Repräsentativität und der Verantwortung der Organisationen der Zivilgesellschaft. Sie müssen ihre neue zentrale Stellung in der Entwicklungszusammenarbeit rechtfertigen. Die Politiker erklären sich insbesondere als einzige ermächtigt, im Namen der Bürger zu sprechen, weil sie demokratisch gewählt worden sind. Den Führungsfiguren der Geschäftswelt kann die Einmischung in ethische Fragen, die möglicherweise den Interessen ihrer Aktionäre oder Kunden widersprechen, ferner ein Dorn im Auge sein. 
Mit der 1989 im Rahmen des Lomé IV-Abkommens eingeführten dezentralisierten Zusammenarbeit wurde ein neuer Ansatz der Entwicklungszusammenarbeit geschaffen. Das Ziel war, mit der Legitimation der Rolle der dezentralisierten Akteure in der Entwicklung den generell eingeschränkten Kreis der Akteure der Entwicklungszusammenarbeit zu erweitern (Fonteneau und Lapeyre, 1998). Die Originalität lag in der Unterstützung der direkt von der Basis ausgehenden Initiativen. Die Verantwortung für die Festlegung der Bedürfnisse und für die Ausgestaltung und Umsetzung des Projekts wurde den lokalen Akteuren übertragen. Diese traten nicht mehr als blosse Empfänger oder Ausführende von auf höherer Ebene festgelegten Projekten, sondern als Antriebskräfte für die Verbesserung ihrer Lebens- und Arbeitsbedingungen auf.

Die Rolle der dezentralisierten Zusammenarbeit als neues Instrument der Entwicklungszusammenarbeit wurde in Art. 12 bis des Lomé-Abkommens neu bekräftigt: „Reconnaissant que les acteurs de la coopération décentralisée peuvent apporter une contribution positive au développement des Etats ACP, les parties contractantes conviennent d'intensifier leurs efforts visant à encourager la participation des acteurs ACP et de la Communauté aux activités de coopération" (In Anerkennung der Tatsache, dass die Akteure der dezentralisierten Zusammenarbeit einen positiven Beitrag an die Entwicklung der AKP-Staaten leisten können, kommen die Vertragsparteien überein, ihre Bemühungen um die Förderung der Beteiligung der AKP-Akteure und der Gemeinschaft an den Zusammenarbeitstätigkeiten zu intensivieren).

Hauptziel der dezentralisierten Zusammenarbeit war es, mit der Errichtung neuer Formen von Partnerschaften zwischen den nationalen und lokalen Behörden, dem Privatsektor und den Organisationen der Zivilgesellschaft die Palette der Entwicklungsakteure zu erweitern, um den Bedürfnissen und Prioritäten der Bevölkerung besser zu entsprechen. Der Gedanke war, eine Neudefinition der Rolle und der Verantwortlichkeiten aller Beteiligten im Entwicklungsprozess zu fördern, indem man durch die Verantwortungsübertragung und durch eine Dezentralisierung der Mittel einen Prozess zur Responsabilisierung der dezentralisierten Akteure unterstützt.

Der neue Ansatz der Entwicklungszusammenarbeit wurde von der Europäischen Kommission mit dem im Juni 2000 unterzeichneten Cotonou-Abkommen noch weiter vertieft. Das Abkommen umfasst insbesondere originelle Bestimmungen zur Förderung von partizipativen Ansätzen, welche die Beteiligung der Zivilgesellschaft sowie der Akteure aus Wirtschaft und Gesellschaft gewährleisten sollen. Gemäss den Bestimmungen des Abkommens werden die nichtstaatlichen Akteure informiert und in die Konsultation über die Politiken und Strategien der Entwicklungszusammenarbeit und über die Prioritäten der Zusammenarbeit, insbesondere in den Bereichen, die sie betreffen oder direkt berühren, sowie über den politischen Dialog einbezogen. Daneben erhalten sie finanzielle Ressourcen, um den lokalen Entwicklungsprozess zu unterstützen. Schliesslich werden sie an der Umsetzung von Projekten und Programmen der Entwicklungszusammenarbeit in den sie betreffenden Bereichen beteiligt.

In diesem Rahmen erhalten die nichtstaatlichen Akteure Unterstützung für den Aufbau ihrer Kapazitäten in entscheidenden Bereichen, um ihre Kompetenzen 
zu stärken, besonders was die organisatorischen Fragen, die Vertretung und die Einführung von Konsultationsmechanismen zur Förderung strategischer Allianzen betrifft. Um den Entwicklungsbedürfnissen der lokalen Körperschaften nachzukommen und um alle Akteure der dezentralisierten Zusammenarbeit, die zur autonomen Entwicklung der AKP-Staaten beitragen können, anzuspornen, unterstützt die Entwicklungszusammenarbeit einerseits die Finanzierung lokaler Mikroprojekte, welche ökonomische und soziale Auswirkungen auf das Leben der Bevölkerung haben, und andererseits die Finanzierung der dezentralisierten Zusammenarbeit, besonders wenn die Anstrengungen und die Organisationsmittel der AKP-Staaten und ihrer Amtskollegen der Europäischen Union zusammengelegt werden.

Der andere wichtige Träger für die Beteiligung nichtstaatlicher Akteure an der Entwicklungszusammenarbeit sind die Strategiedokumente zur Armutsverringerung (Poverty Reduction Strategy Papers, PRSP), die Ende 1999 im Anschluss an eine gemeinsame Initiative von IWF und Weltbank eingeführt wurden (World Bank, 2000; IMF, 2003). Es handelt sich um ein neues Instrument mit dem Ziel, den armen Ländern und den Geldgebern dabei zu helfen, die Auswirkungen ihrer gemeinsamen Anstrengungen bei der Armutsbeseitigung zu verstärken. Die PRSP bilden die konkrete Ausdrucksform des neuen Entwicklungsparadigmas in der Zeit nach dem „Konsens von Washington“.

Um Zugang zu den Ressourcen der wichtigsten Geldgeber zu erhalten, muss das Bewerberland immer häufiger ein solches Dokument vorbereiten, das die sozioökonomischen Verhältnisse des Landes, die langfristigen Zielsetzungen hinsichtlich der Armutsverringerung und die Mittel zur Zielerreichung beschreibt. Das originelle Merkmal des Dokumentes besteht darin, dass es sich aus einem Konsultationsprozess mit umfassender Beteiligung der verschiedenen Akteure, und insbesondere der nichtstaatlichen Akteure, ergeben soll. Seit 1999 hat sich das Instrument der PRSP in den armen Ländern weit verbreitet. Ende September 2003 hatten 14 Länder das definitive PRSP abgeschlossen, 32 Länder hatten ein PRSP-Programm lanciert (IMF, 2003). Die Einführung der PRSP bildete eine wichtige Etappe in der Geschichte der Entwicklungszusammenarbeit. Diese Dokumente sollen die Hilfe stärker koordinieren und die Verfahren der Geldgeber besser harmonisieren. Die Dokumente beschreiben die Ziele, Prioritäten und die Abfolgen in der Armutsbekämpfung, auf deren Basis die bilaterale und multilaterale Hilfe organisiert wird.

Die PRSP verfolgen verschiedene Ziele, darunter namentlich die Förderung der Beteiligung der nichtstaatlichen Akteure durch die Einführung einer Reihe von Partizipationstechniken und die Schaffung von Dialog- und Abspracheräumen. Erwähnenswert ist auch die Bildung von internen und externen Partnerschaften auf neuen Grundlagen, die sich aus einer mehr oder minder umfassenden Neuverteilung der Rollen und Verantwortlichkeiten zwischen den verschiedenen Akteuren der Entwicklung ergeben: zum einen zwischen der Regierung, den lokalen Behörden, dem Privatsektor und den Akteuren der Zivilgesellschaft, zum anderen zwischen der Regierung und den internationalen Geldgebern.

Wie die wachsende Zahl von Fallstudien zeigt, wirft die Ausarbeitung der PRSP jedoch Probleme auf (Booth, 2003; Molenaers und Renard, 2002; Piron und Booth, 2004). Unter den auftretenden Problemen ist der paradoxe Charakter des Aneignungsprozesses aufgrund der Tatsache zu erwähnen, dass die Einführung 
des PRSP-Prozesses in jedem Fall auf eine Auflage der internationalen Organisationen zurückgeht. So wird von den armen Ländern verlangt, ein in Washington genau definiertes Verfahren zu übernehmen, dessen Spielregeln die Weltbank und der IWF in einer Reihe von Orientierungsnotizen und technischen Unterlagen festgeschrieben haben. Es handelt sich demnach um ein weitgehend vorgezeichnetes Verfahren, das effektiv eine neue Form von Konditionalität darstellt. Die Bretton-Woods-Organisationen spielen bei der Festlegung der Zielhierarchie und der Auswahl der Politiken weiterhin eine zentrale Rolle. Die Regierung ist zwar formell für die PRSP verantwortlich, aber die Weltbank und der IWF können den Prozess durch die Gewährung von Krediten oder durch Schuldenerleichterungsmassnahmen bekräftigen bzw. entkräften. Dieses Vetorecht bestimmt auch die Art der vorgeschlagenen Politiken. Zudem beschränkt sich die Partizipation in der Regel auf einen Informations- und Konsultationsprozess mit sehr geringem Einfluss auf das definitive PRSP.

Die Fülle der von den Akteuren der Zivilgesellschaft eingebrachten Empfehlungen wird meistens ignoriert. Schliesslich steht auch nicht die gesamte Armutsbekämpfungsstrategie für eine Beteiligung der Akteure der Zivilgesellschaft offen, denn das prioritäre Ziel - Anpassung an die Normen der globalisierten Wirtschaft - wird keinesfalls zur Diskussion gestellt. Deshalb ist zu beobachten, dass die PRSP nicht alternativen Entwicklungsstrategien die Bahn geebnet haben, und im Gegenteil betreffend den Inhalt den früheren Strukturanpassungsprogrammen weiterhin sehr ähnlich sind, auch wenn sich die Form geändert hat und jetzt partizipative Verfahren einschliesst (Lapeyre und Yépez, 2005). Die grossen Gewinner der PRSP sind in diesem Zusammenhang die Akteure der Privatwirtschaft, die durch ihre aktive Beteiligung an den partizipativen Instrumenten im Rahmen des Prozesses zur Ausarbeitung der PRSP als Akteure der Entwicklung legitimiert werden. Da die RPSP auf dem Gedanken der zentralen Funktion des Wachstums und der Marktkräfte als Wachstumsmotor beruhen, gewinnt die Rolle der Akteure des Privatsektors mit der Förderung von Politiken zugunsten der Marktkräfte, der Akkumulation und der öffentlich-privaten Partnerschaften an Bedeutung.

\section{Bedeutung der PRSP für die Volksorganisationen}

Bei den PRSP erfolgt die Partizipation der Akteure im Rahmen der Zielsetzungen des Wachstums und der Liberalisierung der Akkumulationsbedingungen, wie sie von den Eliten, die das Projekt der Integration in die Weltwirtschaft betreiben, festgelegt wurden (Peemans 2002 : 311). Es handelt sich dabei um eine unantastbare Gegebenheit, und die Partizipation kann demnach nur bessere Information bedeuten, um die Zustimmung der Bevölkerung zu von den Eliten zuvor definierten Zielen zu erlangen. Das Paradoxon des neuen Ansatzes der Bretton-Woods-Institutionen besteht darin, dass sie zwar angeblich die Armen zu Wort kommen lassen, gleichzeitig aber nicht gestatten, dass deren Forderungen und Initiativen von der von ihnen sorgfältig vorgezeichneten Spur abweichen (Lapeyre, 2002).

Der strategische Charakter der PRSP zeigt sich darin, dass die Förderung der neuen öffentlich-privaten Partnerschaften - und damit eine wichtigere Rolle der multinationalen Konzerne - darauf ausgelegt ist, bei der Verwaltung der wesent- 
lichen Dienste eine ökonomische und technische Logik (mit Priorität für die Konzepte Kosten und Effizienz) zu Lasten anderer, von anderen Akteuren befürworteten Logiken einzuführen. Die ansatzweise Integration der Basisorganisationen in die von den Bretton-Woods-Institutitionen festgelegten Partizipationsinstrumente hat zum Ziel, die Organisationen auf Neuland zu locken, ihre Funktionslogik zu ändern und ihre Widerstände zu überwinden. Das Partizipationsinstrument zu übernehmen bedeutet in erster Linie, die Spielregeln und die Machtverhältnisse zu akzeptieren. Das Kräfteverhältnis zeigt sich nicht länger nur im Erteilen von Befehlen, sondern beinhaltet neu auch Überzeugungs-, Informations- und Ausbildungsarbeit mit dem Ziel, die Analyse einer Situation mit Gruppen von Akteuren zu teilen und sie in eine neue Aktions- und Reaktionswelt zu führen. In dieser neuartigen Kontrollregelung zeigt sich eine Weiterentwicklung der Machtausübung durch die dominierenden Akteure (Reynaud, 1997).

In den PRSP realisiert sich ein Diskurs dominierender Akteure, welche die verschiedenen Machtebenen neu gestalten wollen, um die Akzeptanz einer gewissen Anzahl lokaler und nationaler Akteure für die zuvor ausgewählten Ziele im Rahmen des Integrationsprojekts sicherzustellen und so deren Legitimität zu erhöhen. Die PRSP sind der Ausdruck des Kräfteverhältnisses, das auf die Logik realer Akteure zurückzuführen ist. Die Förderung der öffentlich-privaten Partnerschaften ist als Bestandteil einer globalen Strategie der dominierenden Akteure zu sehen, mit dem Ziel, die Akkumulationsbedingungen in der globalisierten Wirtschaft zu fördern und sie von den ihnen auferlegten sozialen Zwängen zu befreien.

Das Machtverhältnis ist jedoch nicht in Stein gemeisselt. Die Einführung von selbst unvollkommenen Partizipationsinstrumenten kann potenziell dazu beitragen, aktive Subjekte auf den Plan zu rufen, die in der Lage sind, die ihnen vorgeschlagenen Initiativen in Frage zu stellen. Dadurch, dass die PRSP den Organisationen der Zivilgesellschaft mehr Informationen und Diskussionsmöglichkeiten anbieten, wird das Kräfteverhältnis zwar nicht grundsätzlich verändert, jedoch werden die verfügbaren Ressourcen neu verteilt. Diese Veränderungen können auch die Beziehungen in den Verhandlungen ändern und den Akteuren aus dem Volk mehr Möglichkeiten an die Hand geben, ihren Zugang zu den wesentlichen Diensten selbst zu garantieren. Unausgesprochen bleibt im funktionellen Partizipationsansatz der Bretton Woods-Organisationen, dass die Initiativbefugnis niemals gleich verteilt ist, und dass man sich ihrer nicht frei bemächtigen kann. Die wirkliche Herausforderung in Bezug auf die Entwicklung ist deshalb die Ownership der Basisorganisationen für das Partizipationsinstrument als solches, um die Spielregeln zu verändern, welche festlegen, wer die Vorschlags- und Initiativbefugnis hat.

\section{Rolle der Basisakteure}

Indem die neuen Partnerschaften und die Partizipation der Zivilgesellschaft in den Vordergrund gerückt werden, können eine ganze Reihe von Akteuren, welche in die Kategorie der passiven „Armen“ abgeschoben worden waren, wieder den Status von Akteuren der Entwicklung erhalten. Schliesslich bedeutet dies, die Augen für eine andere Realität der Entwicklung zu öffnen, die von einer 
zunehmenden Anzahl von Arbeiten enthüllt wird, welche die Initiativfähigkeit der Bevölkerung im Alltag angesichts der Krise von Wirtschaft und Staat zeigen (Peemans, 2002, Scott, 1985, Monnier und Droz, 2005). Die durch die wirtschaftliche Globalisierung bewirkten neuen Formen der Präkarisierung und Ausgrenzung dürfen nicht zum Fazit verleiten, dass die Bevölkerung diesen Zustand passiv erträgt. Gerade in den prekären sozialen Lebensräumen gelingt es immer mehr Personen, ihrem Verhalten neuen Sinn zu geben und wieder Bindungen und einen Austausch mit anderen zu schaffen.

Diese radikale Verlagerung des Blickwinkels zu den „,vergessenen Akteuren“ das heisst denjenigen, die lange als Nichtakteure der Entwicklung angesehen wurden - muss zu einer völlig anderen Konzeption der aktuellen Herausforderungen der Entwicklungspolitik und mithin der Entwicklungszusammenarbeit führen. Es ist in der Tat festzustellen, dass zwischen den Entwicklungswünschen der Bevölkerung, wie sie sich in der Vielzahl der volkstümlichen Praktiken offenbaren, und den von den dominierenden Akteuren festgelegten Entwicklungsprioritäten häufig eine tiefe Kluft besteht.

Die Armenviertel der Grossstädte im Süden bilden zum Beispiel keinen Ort sozialer Desorganisation und mangelnder Ordnung, sondern einen Lebensraum, in dem Armut und Ausgrenzung die Aktion nicht ausschliessen. Auch Bevölkerungsgruppen in prekären Lebensverhältnissen sind in der Lage, sich kollektiv zu mobilisieren, um zu handeln und die soziale Bindung, auf der die Sicherung ihrer Lebensbedingungen beruht, ständig neu zu erfinden. Ihre Eingliederung in das Sozialgefüge und die Mitbestimmung bei der Gestaltung ihres Lebensraumes äussern sich auf unterschiedliche Weise und werden von kultureller und sozialer Vielfalt geprägt. Die Fähigkeit dieser Akteure, die Welt und ihre Entwicklungsprojekte entsprechend ihrer kulturellen Identität, ihrer Geschichte und ihrem Lebensraum zu konzipieren, muss somit anerkannt werden. In diesen Praktiken der Basis, die man als ,,volkstümlich“ bezeichnen könnte, verbinden sich individuelle Strategien, Netzbildungen und mehr oder minder weit entwickelte Vereinigungen.

Die Anerkennung der Vielfalt der Entwicklungsakteure führt unweigerlich zur Anerkennung der Vielfalt der Lösungen des Problems betreffend den Zugang zu den wesentlichen Diensten. So wirkt sich auch die aktuelle Privatisierungstendenz sehr unterschiedlich aus, je nachdem, ob im Interesse der multinationalen Konzerne oder im Interesse der kleinen lokalen Unternehmen privatisiert wird. Im Süden hat der informelle Sektor seit den 80er Jahren stark an Bedeutung gewonnen, weil die Bevölkerung diesen Sektor braucht, um den immer unsicheren Lebensbedingungen und dem Rückzug des Staates standzuhalten. Im informellen Sektor spielen sich die vielfältigen volkstümlichen Wirtschaftspraktiken $\mathrm{ab}$, die den Lebensraum der Bevölkerung strukturieren und ihr, mehr schlecht als recht, Einkommen, Beschäftigung und Zugang zu den wesentlichen Dienstleistungen sichern. Anders jedoch als die multinationalen Konzerne sind die lokalen Kleinproduzenten im lokalen „kleinen Markt“ verankert. Dabei handelt es sich um einen richtigen Markt, der aber fest in den gesellschaftlichen Realitäten verwurzelt ist und durch die Kombination von Marktlogik, Umverteilung und sozialer Bindung geprägt ist (Peemans, 2002 : 386). Die Akkumulation ist dort noch starken sozialen Regelungen unterworfen, zusammen mit der (Inter)Dependenz-Beziehung, welche die Bevölkerung und die lokalen Klein- 
produzenten verbindet. Solche privaten Anbieter $\mathrm{zu}$ unterstützen, bedeutet somit, bestimmte Wirtschaftspraktiken zu fördern, die nicht ausschliesslich von der Akkumulationslogik beherrscht sind, und ein anderes Entwicklungsmodell als das von den multinationalen Konzernen und den transnationalen Eliten propagierte Modell zu fördern.

Die Bevölkerung ist angesichts der Krise des öffentlichen Sektors im Süden nicht untätig geblieben, sondern hat sich mobilisiert, um ihren Lebensrahmen abzusichern. In diesem Zusammenhang waren interessante Beispiele für die „Privatisierung von unten“ (im Gegensatz zur „Privatisierung von oben“, die von den nationalen und internationalen Eliten beschlossen wird), zu beobachten. Dabei handelt es sich um echte kollektive Initiativen in den Bereichen Wasser, Strom, Wohnungswesen oder Basisgesundheitsversorgung. Sehr aufschlussreich ist in diesem Zusammenhang das Beispiel des dahoulagel in Bukavu, Demokratische Republik Kongo (Muhinduka, 2004). Wegen der Beeinträchtigung des Versorgungsnetzes der nationalen Stromgesellschaft (Société nationale d'électricité, SNEL) kam es in den 90er Jahren in den einfachen Wohnvierteln von Bukavu immer häufiger zu Stromabschaltungen und Stromversorgungsproblemen. Vor diesem Hintergrund wurden kollektive Praktiken entwickelt, um den Akteuren des Volkes den Stromzugang zu garantieren. Dahoulage stellt eine Reaktion des Volks auf die Frage des Zugangs zu einer wesentlichen Dienstleistung für die benachteiligten Gesellschaftsschichten dar.

Da die potenziellen Verbraucher in den ärmeren Vierteln wenig solvent und ausserstande sind, die monatlichen Ausgaben und die individuellen Anschlusskosten zu zahlen, wird die SNEL diesen Gruppen sehr wahrscheinlich keinen Zugang zu ihrer Dienstleistung gewähren. In diesem Kontext organisieren sich lokale Gemeinschaften in bestimmten Quartieren; ihre Mitglieder legen Geld zusammen, um einen Haushalt ans Netz anzuschliessen. Von diesem Haus aus werden Stromleitungen gezogen, um die verschiedenen Mitglieder, die einen Beitrag geleistet haben und die je nach Stromverbrauch bezahlen, mit Strom zu versorgen. So entstehen Gemeinschaftsorganisationen, die sich um Abonnements kümmern, aber auch in zahlreichen Streitfällen als „Strom-Polizisten“ walten, mit den Beamten der SNEL über die Stromumleitung feilschen und verhandeln und sich für dahoulage einsetzen. Mit der Verlängerung der dahoulage-Kette sinkt jedoch die Stromstärke ab und es kommt zu einer Überhitzung der Transformatoren. Die lokalen Gemeinschaften nehmen dann die Verwaltung dieser wesentlichen Dienstleistung in die Hand. Sie legen Geld zusammen, um einen leistungsfähigeren Transformator zu kaufen, und handeln mit der SNEL ein Verhandlungsprotokoll aus, das ihnen den Stromzugang gewährleistet. So zeichnen sich an der Basis andere Partnerschaftsformen ab, welche die soziale Bindung neu erfinden und damit Teil einer echten Entwicklungsdynamik werden.

Dahoulage ist ein in der Stadt Bukavu geläufiger Neologismus. In der lokalen Kultur bedeutet der Begriff freie und obligatorische Versorgung mit Feuer beim Nachbarn für Haushalte, die kein Feuer haben. 
Die Idee der öffentlich-privaten Partnerschaften und der Beteiligung nichtstaatlicher Akteure an der Verwaltung wesentlicher Dienstleistungen hat in den 90er Jahren allmählich in die Entwicklungszusammenarbeit Einzug gehalten. Wie erwähnt, beruhen diese Partnerschaften aber auf einer sehr unterschiedlichen Logik: Akkumulationslogik, wenn bevorzugt auf die Betreiber des ,grossen Marktes" - sprich die multinationalen Konzerne - gesetzt wird, Logik der sozialen Bindung, wenn die Volksorganisationen zum Tragen kommen, aber auch gemischte Logik im Fall der lokalen Betreiber des kleinen Marktes, deren Wirtschaftspraktiken in soziale Strukturen eingebettet sind, die sich in einem instabilen Gleichgewicht befinden und ständig neu definiert werden.

Die jüngste Anerkennung der Bedeutung der Partizipation zeigt, dass die Vielfalt der Entwicklungsakteure und ihrer Strategien besser erkannt wird. Zu den Hauptgefahren aller Initiativen des Wandels gehört nicht nur Revolte oder aktiver Widerstand, sondern auch Gleichgültigkeit und Distanz der Zielbevölkerungen, die den Initianten mit grosser Zurückhaltung begegnen. Um die Bevölkerung zu echten Partnerschaften für die Verbesserung ihrer Lebens- und Arbeitsbedingungen zu mobilisieren, sollte ein institutionelles Umfeld mit einem angemessenen Rahmen für die Verhandlung und die Erreichung eines Kompromisses um ein globales Projekt der Entwicklungszusammenarbeit errichtet werden; so kann sich eine kollektive Aktionsfähigkeit heranbilden, welche kollektives Lernen und soziale Bindung ermöglicht. Ein solcher um die Partizipation bereicherter Ansatz muss in einem globaleren Demokratievorhaben verankert werden, welches das Recht der Gemeinschaft auf eigene Ausdrucksmöglichkeiten und auf Selbstbestimmung hinsichtlich ihres Entwicklungsprojektes am Ort, wo sie lebt, garantiert.

Der vorliegende Artikel hält es nicht mit der konfliktlosen Vision der Beteiligten (Stakeholders), welche den vorherrschenden Diskurs über die neuen Partnerschaften prägt, sondern er versucht die Notwendigkeit aufzuzeigen, den zentralen Stellenwert des Konflikts, der Verhandlung und des Kompromisses anzuerkennen. Das Konfliktkonzept ermöglicht es, den kollektiven Akteur zu entdecken, der sein Projekt erläutert, die Standpunkte gegenüberstellt, sich mit anderen Akteuren auseinandersetzt, sich um Einigung und Kompromisse bemüht, seine Autonomie und seinen Handlungsspielraum wahren möchte. Der Konflikt ist eine normale Funktionsweise; Konflikte zwingen dazu, durch die Verhandlung eine gemeinsame und gegenseitig akzeptable Definition des Konfliktgegenstandes zu finden. Das ist eine unerlässliche Voraussetzung für die Einführung echter Partnerschaften.

Die sozialen Institutionen müssen sich zwar weiter entwickeln und sich dem neuen Druck der globalisierten Wirtschaft anpassen, aber der Staat bleibt in diesem Spiel der Vermittlung, das die Erhaltung der sozialen Bindung erlaubt, ein zentraler Akteur. Wenn sich private Interessen den institutionellen Formen sozialer Kontrolle entziehen, zerstören sie den sozialen Zusammenhalt. Diese Tendenz hatte den Staat im modernen kapitalistischen System veranlasst, aktiv in den sozialen Bereich einzugreifen. Die Suche nach einem ,dritten Weg“ als eine der zahlreichen Varianten der demokratischen Gesellschaft mit einer gemischten Wirtschaft setzt eine Neudefinition der Rolle des Staates voraus, welche die Frage: 
„welcher Staat und wozu“ nach sich zieht. Die Frage des Zusammenhangs zwischen Markt, Staat und Zivilgesellschaft bildet den Kern der Reflexion über tragfähige alternative Entwicklungsprojekte. In diesem Rahmen stellt die Erhaltung des sozialen Bindeglieds eine grundlegende Entwicklungsdimension dar.

Institutionelle Innovationen sind wesentlich, um die vielen volkstümlichen Praktiken zu unterstützen, die überall im Süden die Lebensverhältnisse derjenigen zu sichern versuchen, die von den Integrations- und Strukturanpassungspolitiken aussen vor gelassen wurden. Allerdings muss der verklärende Ansatz der lokalen Initiativen überwunden werden, um zu verstehen, welche der vielfältigen Praktiken des Widerstands, des Überlebens, der Solidarität und der Absicherung der Lebensverhältnisse zu einem anderen Modell des ,Zusammenlebens und zusammen Handelns" führen können. Diese Praktiken existieren zwar, jedoch werden damit die Prekarisierung und die Ausgrenzung, unter denen die Bevölkerung der betroffenen Quartiere nach wie vor leidet, nicht aufgehoben. Es ist zu betonen, dass die Unterwerfung oder das Gewicht externer Sachzwänge ihre Initiativfähigkeit nicht schwächen. Diese Akteure wollen lokale Initiativen entwickeln können, ihre eigenen Aktionsregeln festlegen oder den Ort ihres Lebens mitbestimmen können. Sie versuchen, Autonomie auszuüben, indem sie Handlungsspielräume ausnutzen oder indem sie verhandeln; der aktuelle Kontext bietet ihnen neue Möglichkeiten zur Verwirklichung ihrer Projekte.

\section{Bibliographie}

Booth, D., 2003, Introduction and Overview, Development Policy Review, Vol. 21, No 2.

Camdessus, M., 2003, Report of the World Panel on Financing Water for Infrastructure, Water for All, Kyoto, World Water Council.

Cardoso, F., 2004, Nous, peuples: société civile, Organisations des Nations Unies et gouvernance mondiale, Rapport du Groupe de personnalités éminentes sur les relations entre l'Organisation de Nations Unies et la société civile, New York, Nations Unies.

Colclough, C., 1992, „Who Should Learn to Pay?“, in C. Colclough and J. Manor, (eds.), States or Markets, London, Clarendon Press.

Coleman, J., 1990, Foundations of Social Theory, Harvard, Harvard University Press.

Commission européenne, 2000, L'accord de Cotonou entre les pays ACP et la Communauté européenne, Brüssel, Europäische Kommission.

Cornia, G., Jolly, R., Stewart, F.,1987, Adjustment with a Human Face, Oxford, Oxford University Press.

Evans, P., 1995, Embedded Autonomy, Princeton, Princeton University Press.

Evans, P., 1996, „Government Action, Social Capital and Development: Reviewing the Evidence on Synergy“, World Development, Vol. 24, No 6.

Fukuyama, F., 1995, Trust - The Social Virtues and the Creation of Prosperity, London, Hamish Hamilton.

Hopkins, M., 2004, Corporate Social Responsibility: an Issues Paper - Working Paper No 27, Geneva, ILO (International Labour Organization).

IMF, 2003, PRSPs: Detailed Analysis of Progress in Implementation, Washington, D. C., International Monetary Fund.

Isham J., Narayan, D., Pritchett, L., 1995, „Does Participation Improve Performance? Establishing Causality with Subjective Data“, World Bank Economic Review, Vol. 9, No 2.

Jolly, R., Emmerij, L., Ghai, D., Lapeyre, F., 2004, UN Contributions to Development Thinking and Practice, Bloomington, Indiana University Press.

Kaul, I., Grunberg, I., Stern, M., 1999, Global Public Goods, Oxford, Oxford University Press.

Krueger, A., 1990, „Economists’ Changing Perceptions of Government“, Weltwirtschaftliches Archiv, Band 126, Heft 3. 
Lapeyre F., 2002, „Le rêve d'un développement sans conflit“, Nouveaux Cahiers de l'IUED, No 13, Paris, PUF.

Lapeyre, F. Fontenau, G., 1998, Les organisations syndicales et la coopération décentralisée, Brüssel, Europäischer Gewerkschaftsbund.

Lapeyre, F., Yépez, I., 2005, „Les processus participatifs dans les Documents Stratégiques de Réduction de la Pauvreté (DSRP): Quelles perspectives pour les pratiques populaires“, in C. Mainguy, H. Gerardin, J. Brot et G. Froger, Quels acteurs pour quel développement, Paris, Karthala.

Moelnaers, N., Renard, R., 2002, „Strengthening Civil Society from Outside? Donor-driven Consultation and Participation Processes in Poverty Reduction Strategies: the Bolivian case“, Working Paper, No 10/2002, Antwerp, Institute of Development Policy and Management, University of Antwerp.

Monnier, L., Droz, Y., (dir.), 2004, „Coté jardin - Coté cour - Anthropologie de la maison africaine, Nouveaux Cahiers de l'IUED, No 15, Paris, PUF.

Muhinduka, D., 2004, Les organisations communautaires et l'entreprise publique d'électricité SNEL: la fourniture de l'électricité à Bukavu de 1990 à 2004 - mémoire de DEA, Louvain, Université Catholique de Louvain.

Peemans J-P., 2002, Le développement des peuples face à la modernisation du monde, Paris, Academia, L'Harmattan.

Peemans, J.-P., 1997, „Les enjeux d'une réflexion sur la décentralisation et le développement local en Afrique au Sud du Sahara: quelques remarques introductives“, papier présenté au Colloque „Les dimensions sociales et économiques du développement local et la décentralisation en Afrique au Sud du Sahara, 20-21 novembre, Louvain-la-Neuve.

Piron, L.H., Booth, A., 2004, „Politics and the PRSP Approach: The Bolivian Case Study“, Working Paper, No 238, London, Overseas Development Institute.

Putnam, R., 1993a, Making Democracy Work: Civic Tradition in Modern Italy, Princeton, Princeton University Press.

Putnam, R., 1993b, „The Properous Community“, American Prospect, no.13.

Reynaud, J.-D., 1997, Les règles du jeu, Paris, Armand Colin.

SAPRIN (Structural Adjustment Participatory Review International Network), 2004, Structural Adjustment, London, Zed Books.

Scott, J., 1985, Weapons of the Weak, New Haven, Yale University Press.

Stiglitz J., 1998, Towards a New Paradigm for Development, $9^{\text {th }}$ Raúl Prebisch Lecture, Geneva, UNCTAD.

Tinbergen, J., 1984, „Development Cooperation as a Learning Process“, in G. Meier and D. Seers, (eds.), Pioneers in Development, Oxford, Oxford University Press.

Tollison, R., 1982, „Rent Seeking: A Survey“, Kylos, Vol. 35, Fasc. 4.

UNDP (United Nations Development Programme), 1990, Human Development Report, New York, UNDP.

UNDP, 2003, Human Development Report 2003 - Millennium Development Goals, New York, UNDP.

United Nations, 2004, Report of the Secretary-General in Response to theReport of the Panel of Eminent Persons on United Nations-Civil Society Relations, A/59/354.

Wolfensohn, J., 1997, Adress to the 7th Meeting of the International Labour Conference, $85^{\text {th }}$ Session, $12^{\text {th }}$ June, Provisional Record 11, Geneva, ILO.

Wolfensohn, J., 2000, „Building an Equitable World“, Address to the Boards of Governors, Prag, Tschechische Republik, 26. September.

Woolcok, M., 1998, ,Social Capital and Economic Development: Toward a Theoretical Synthesis and Policy Framework", Theory and Society, Vol. 27.

World Bank, 1981, Development in Sub-Saharian Africa, Washington D.C., World Bank.

World Bank, 1990, Poverty - World Development Report, Washington D. C., World Bank.

World Bank, 1998, Assessing Aid: What Works, What doesn't, and Why, World Bank Policy Research Report, Oxford, Oxford University Press.

World Bank, 2000, Attacking Poverty - World Development Report, Washington D. C., World Bank. 